\title{
e-Phaïstos
}

e-Phaïstos

Revue d'histoire des techniques / Journal of the history

of technology

II-1 | 2013

Savoirs et sciences sur les machines

\section{L'école des producteurs. Activités économiques et institutionnalisation de la formation au travail dans la région du Nord, des années 1860 aux années 1930}

The School of Producers

Stéphane Lembré

\section{(2) OpenEdition}

Journals

Édition électronique

URL : http://journals.openedition.org/ephaistos/1350

DOI : 10.4000/ephaistos. 1350

ISSN : 2552-0741

Éditeur

IHMC - Institut d'histoire moderne et contemporaine (UMR 8066)

Édition imprimée

Date de publication : 1 juin 2013

Pagination : 111-116

ISSN : 2262-7340

Référence électronique

Stéphane Lembré, «L'école des producteurs. Activités économiques et institutionnalisation de la formation au travail dans la région du Nord, des années 1860 aux années 1930 », e-Phaïstos [En ligne], II-1 | 2013, mis en ligne le 15 décembre 2019, consulté le 15 septembre 2020. URL : http:// journals.openedition.org/ephaistos/1350 


\title{
Stéphane Lembré
}

\section{L'école des producteurs. Activités économiques et ins- titutionnalisation de la formation au travail dans la ré- gion du Nord, des années 1860 aux années 1930}

\author{
Thèse de doctorat, Université Charles-de-Gaulle - Lille 3 \\ Institut de recherches historiques du Septentrion (IRHiS) \\ Soutenue le 16 septembre 2011 \\ 2 vol., 765 p.
}

Directeur de thèse :

Jean-François CHANET, professeur, Sciences Po

Jury:

Anne-Françoise GARÇON, professeur, Université Paris I (rapporteur et présidente)

Gérard BODÉ, chargé de recherches, Institut français de l'éducation

Jean-François ECK, professeur émérite, Université Lille 3

André GRELON, directeur de recherches, EHESS (rapporteur)

Paul-André ROSENTAL, professeur, EHESS et Sciences Po
Madame la Présidente,

Messieurs les membres du jury,

Le travail de thèse que j'ai le plaisir et l'honneur de soutenir sous le titre L'école des producteurs. Activités économiques et institutionnalisation de la formation au travail dans la région du Nord des années 1860 aux années 1930 est né, comme bien des démarches scientifiques, d'une volonté de discuter, de questionner l'évidence : en l'occurrence, celle du développement continu et nécessaire de la formation. En des temps où la formation continue, la formation permanente ou la formation tout au long de la vie sont considérées comme les outils d'un bon management, où la formation initiale est jugée déterminante pour entrer avec quelques atouts sur le «marché » du travail, il me semblait et il me semble toujours utile de revenir sur ce besoin de former et de se former. D'une certaine façon, ce besoin est aujourd'hui présumé, voire revendiqué comme une conséquence salutaire des difficultés économiques que connaissent les sociétés des pays dits développés depuis les années 1970. Le dogme de la croissance économique est alors basé sur la nécessité de reconvertir des économies industrialisées concurrencées pour les productions de masse vers des professions et des productions qui requièrent un savoir-faire spécifique. 
Les enseignements techniques trouveraient donc naturellement leur utilité, à condition d'être centrés sur la pratique plus que sur la théorie, de tenir compte aussi des besoins du "marché » du travail, de fournir enfin des personnels « prêts à l'emploi ». Autant d'enjeux qui ne datent assurément pas d'aujourd'hui. Mais si l'on retrouve des débats comparables quant à la part respective de l'enseignement pratique et de l'enseignement théorique, ou de l'adaptation aux besoins économiques locaux, il faut également souligner de profondes différences entre notre présent et ce passé, à commencer par celle qui concerne la reconnaissance des besoins de formation. La constitution d'enseignements techniques initiaux, distincts de l'instruction élémentaire et du début de l'activité professionnelle, correspond à des évolutions sociales et économiques importantes, ainsi qu'à la construction complexe de besoins de formation.

Sur la base d'une définition de la formation au travail rassemblant les enseignements techniques industriels et commerciaux, les enseignements agricoles, l'apprentissage et toute institution délivrant des savoirs et savoir-faire initiaux à visée professionnelle dans les domaines industriels, commerciaux et agricoles, c'est d'abord à la compréhension de cette construction que vise ce travail, à partir des réalisations et des discussions des différents acteurs de cette histoire, et pour une période de foisonnement des initiatives. Cette période s'étend des années 1860 , dans le sillage du traité de commerce franco-britannique, jusqu'à la crise des années 1930 qui entraîne, avant la Deuxième Guerre mondiale, une modification profonde de la manière d'envisager la formation et des moyens mis en œuvre. La fin des années 1870, avec l'installation des républicains au pouvoir et la mise en place progressive de nouvelles structures pour l'enseignement technique, puis la Première Guerre mondiale, qui fait passer des appels multipliés à la réforme à la refonte d'un système, marquent à l'évidence des étapes dans l'institutionnalisation des formations. Les trois périodes ainsi délimitées se distinguent nettement par l'importance qu'y acquièrent les besoins de formation et par la place qu'y prennent les institutions de formation.

Ce travail s'inscrit dans une veine historiographique attachée à décrire et comprendre le fonctionnement des institutions de l'économie. À la suite de travaux qui ont décrit les missions et le fonctionnement de chambres de commerce, ou qui ont scruté les solidarités familiales et financières du capitalisme nordiste, il me paraît essentiel d'aborder un thème, la formation au travail, qui se place à l'intersection des intérêts privés et de l'action publique, et qui sonde à nouveaux frais l'attachement aussi fort qu'ambigu au libéralisme patronal. Parmi les équipements nécessaires à l'activité économique, aux côtés des infrastructures matérielles, la qualification des hommes et des femmes est un objet assurément complexe, qui oscille d'abord entre le déni et la reconnaissance. Il s'agit aussi, ce faisant, de revenir sur le paternalisme et la prise en compte de ce type de formation, alors que le rôle des grands patrons paternalistes est mieux connu pour l'éducation élémentaire. Enfin, comment les entrepreneurs, individuellement et collectivement, se sont-ils engagés dans la formation au travail ou, au contraire, ont pu être réticents, avec des motifs d'ailleurs variés dans l'un comme dans l'autre cas ? À partir de ces questionnements, les sources mobilisées ont été aussi bien celles des chambres de commerce, d'une grande richesse, que celles d'entreprises et de syndicats de différentes envergures.

Du côté de l'histoire de l'éducation, au sein de laquelle l'histoire des formations au travail est longtemps restée dans l'ombre, des avancées ont été permises par la prise en compte de l'offre locale d'enseignement et par des monographies importantes, individuelles ou collectives, sur Nancy, sur Nantes ou sur Lyon, pour n'en citer que trois parmi les plus importantes. Le questionnement des systèmes scolaires nationaux et de leur relation au local, comme la réflexion - difficile - sur les espaces européens du savoir, sont également bien 
engagés, grâce à des études aussi bien monographiques que centrées sur les étudiants ou les professeurs par exemple. L'approche institutionnelle, à travers des études exemplaires sur l'enseignement primaire supérieur, fournit aussi des schémas d'analyse utiles. Néanmoins la tendance au morcellement des travaux et des méthodes employées met au défi de proposer un cadre d'analyse pertinent. Or, envisager l'institutionnalisation des formations au travail par sa dimension territoriale me paraissait important, afin d'identifier des logiques, des institutions développées en réseau, à l'image des écoles pratiques, et aussi la hiérarchisation des institutions. De plus, cette approche facilitait l'identification des circulations réelles, souhaitées ou supposées de références, d'élèves et de personnels.

Il s'agissait au total de repérer et d'expliquer comment a fonctionné la construction d'un système régional de formation - à supposer même que l'on puisse évoquer un système, ce qui, au sens fermé et stable du terme, est probablement illusoire. Pour y parvenir, plusieurs choix se sont imposés quant à la délimitation du corpus et à la méthode.

Le premier choix est inhérent à l'objet même de ce travail, à savoir la profusion et la grande hétérogénéité des dispositifs de formation, dans l'espace et dans le temps. Il était toutefois exclu de prétendre à un répertoire complet. Un tel inventaire, qui devrait aussi prendre en compte la richesse des enseignements professionnels de l'Ancien Régime et du premier XIX ${ }^{\mathrm{e}}$ siècle, est déjà largement engagé pour le département du Nord grâce à Philippe Marchand, mais il reste à faire pour le Pas-de-Calais.

Saisir des dynamiques et un mouvement d'institutionnalisation supposait de prendre en compte, derrière la variété des archives consultées, la diversité des expériences - une diversité qu'il fallait éviter de réduire sous prétexte de proposer un cadre intelligible. D'où le choix, délibéré, d'envisager ensemble des formations industrielles et commerciales (souvent créées ou développées en lien les unes avec les autres) ainsi que les forma- tions agricoles et artisanales. Tout en étant bien conscient de la spécificité de ces différentes formations, des publics visés et des projets sociopolitiques dont elles émanent, je souhaitais plutôt montrer comment, à partir de cette diversité même, a pu se construire - de manière sans doute inachevable et en tout cas inachevée dans les années 1930 - un système régional de formation. Pour prendre la mesure de cette particularité supposée, il fallait relever, par-delà la diversité, des points communs dans la région, depuis l'intérêt des conseils généraux et municipaux pour ces questions jusqu'à l'attention portée aux pratiques extérieures de formation, en France ou à l'étranger.

Car dans le même temps, la spécificité nordiste supposée doit beaucoup - et ce n'est pas contradictoire - à la comparaison internationale, aux leçons prises à l'étranger. Le rôle de passeurs et le thème du besoin de formation fonctionnent également de manière transversale. La construction de la « spécificité nordiste » se nourrit de l'emboîtement des échelles, du local au transnational. Autant d'éléments qui plaident pour une approche globale, dont la nécessité tient aux caractéristiques économiques et sociales du Nord de la France, et en particulier la coexistence d'une agriculture très active et de dynamiques industrielles, minière et textile.

Ces observations rejoignent la réserve née de l'observation de cheminements historiographiques distincts qui paraissent postuler une frontière hermétique entre deux mondes dont on sait pourtant bien qu'ils n'étaient pas aussi étanches. Il n'est pas rare, durant la période considérée, que ces enseignements techniques commerciaux, industriels et agricoles soient envisagés ensemble, abordés avec des préoccupations comparables, voire même parfois comparés entre eux. Ce n'est pas nier les caractéristiques propres aux espaces ruraux et aux espaces urbains, que d'en montrer les relations permanentes.

Tout comme le projet n'est pas celui d'un inventaire des enseignements techniques dans la région du Nord, il ne correspond nullement à l'étude d'un 
isolat régional, mais plutôt à la saisie de la construction continuée du système de formation à partir des dispositifs mis en place sur le terrain. Bien sûr, il s'agit de l'étude d'une région puissamment industrielle et agricole, aux dynamismes démographique et économique forts tout au long de la période envisagée. Mais la question des spécificités ou, au contraire, des points communs par rapport à ce qui se passe ailleurs en France n'offre pas toujours des réponses fermes, faute le plus souvent de travaux du même type. L'échelle régionale devait être testée comme un échelon intermédiaire progressivement investi par les principaux promoteurs des formations d'une fonction régulatrice. À cet égard, l'étude porte tout autant sur les territoires locaux de la formation que sur les circulations transnationales de références, et envisage la région du Nord avec l'ambition de dégager des perspectives qui dépassent ce cas. La région est érigée en espace d'observation des emboîtements territoriaux et des dynamiques qui ont animé l'histoire des formations au travail.

Le dépouillement de sources nombreuses a permis d'identifier rapidement et de se confronter à trois types de difficultés.

La question de la circulation, liée à celle des comparaisons, a requis une longue réflexion et des lectures multipliées pour comprendre de quels modèles les promoteurs d'institutions de formation nordistes se revendiquent, et les décalages éventuels (en réalité fréquents) entre le modèle invoqué et les emprunts effectifs. Finalement, j'ai choisi d'inclure cet aspect dans celui de l'emboîtement des échelles d'analyse, et donc d'articuler trois niveaux constamment changeants et en interaction, de la revendication d'adaptation locale des formations à la circulation des références et des modèles pédagogiques. Sans méconnaître le rôle des administrations centrales et de leurs représentants, l'étude précise de certains cas conduit à nuancer à la fois l'idée d'adaptation locale des formations, dont l'importance n'exclut pas l'action décisionnaire régionale ou nationale, et celle de circulation, bien plus souvent revendiquée que réellement pratiquée. Le spectre de l'enseignement technique allemand après la guerre de 1870 est le cas le plus emblématique de cette invocation de l'étranger comme aiguillon à la réforme, même s'il est logiquement complété, dans le Nord de la France, par la référence aux formations agricoles et industrielles belges. En nous centrant sur le rôle des passeurs, la réalité et les limites des circulations peuvent être mieux repérées : le cas des responsables catholiques lillois, à la recherche de modèles du côté de Louvain au moment où les catholiques sont au gouvernement en Belgique, montre combien les projets socio-politiques ont leur part dans des circulations, réelles ou revendiquées, qui ne doivent pas grandchose au hasard.

À partir de cet angle d'approche, le statut de la comparaison trouvait une place mieux délimitée. Parmi les réflexions sur la démarche comparative existent en effet des différences sensibles. La présence dans les fonds d'archives consultés de brochures d'institutions de formation étrangères et les compte rendus d'enquêtes plaidaient en premier lieu pour une étude des comparaisons par les promoteurs nordistes de la formation eux-mêmes. Qu'il s'agisse de passeurs, ayant effectivement visité des établissements et rencontré des responsables, ou que les comparaisons soient de seconde main, une fois la documentation rassemblée, la référence à un "ailleurs » est toujours subordonnée aux projets domestiques. Distinguer les emprunts réels de ces références de circonstance implique de redoubler la démarche comparative : cette fois, c'est l'historien qui doit procéder à la comparaison, pour apprécier les similitudes et les divergences. Â défaut de pouvoir envisager ce travail de manière systématique, il a été accompli pour plusieurs cas. Ce double niveau comparatif, choisi de manière empirique au fur et à mesure de la réalisation de la thèse, a l'avantage de placer les comparaisons et les circulations au centre d'un projet qui postulait dès l'origine l'emboîtement des espaces d'institutionnalisation de la formation. Il s'inscrit aussi dans une réflexion critique sur la 
place de l'historien dans l'historiographie du transnational en plein épanouissement.

La deuxième difficulté rapidement apparue, et qui était attendue, est liée à la profusion des dispositifs de formation de toute nature. Comment prendre en compte leur diversité sans se perdre dans le détail ? Il n'était pas possible de s'intéresser en détail aux contenus des enseignements, aussi intéressants soient-ils. La compréhension des logiques d'institutionnalisation nécessitait néanmoins de recourir ponctuellement à des études plus précises des savoirs ou de la sociologie des élèves, ce qui a été fait à travers l'étude des boursiers, pour les inscrits au cours d'hydrographie de Dunkerque ou, dans les annexes, à partir d'annuaires d'anciens élèves. De même, les choix de plan d'études et de dispositifs pédagogiques sont importants pour apprécier le succès d'une formation, mais aussi le positionnement institutionnel souhaité par ses promoteurs et les décalages éventuels entre les intentions et les réalisations.

Le troisième point problématique se trouvait à la rencontre des logiques économiques, sociales et techniques - si l'on peut les distinguer. La question des rapports entre progrès technique et reconnaissance des besoins de formation allait de soi. Le réagencement des qualifications induit par l'introduction de la machine dans le processus de production est un moment évidemment important. Mais il importe d'étudier de très près ces évolutions, dont les rythmes et les modalités précises sont très variables : la formation se trouve dès lors comme enchâssée dans de multiples interactions entre modes de production, décisions politiques et réformes sociales. La «moralisation » des classes laborieuses, si souvent invoquée, a sans doute fonctionné souvent comme un prétexte, mais elle ne paraît pas contradictoire d'objectifs sociaux réels, à commencer par le contrôle et la fixation de populations, ou la socialisation de celles-ci. Le développement de la législation sociale au tournant du XIXe et du XXe siècle a eu des conséquences directes sur la formation au travail. Certains observateurs ou ac- teurs économiques n'ont pas hésité à lui imputer la «crise de l'apprentissage ». Il fallait déconstruire les logiques à l'œuvre, envisager la technique comme fait humain et détailler la compatibilité recherchée entre objectifs économiques et motivations sociales : tout cela justifiait une approche des expériences de formation sur le terrain, pour mieux les confronter aux différents discours tenus. La même prise en compte de multiples logiques était aussi nécessaire pour suivre l'établissement des critères pour la reconnaissance des diplômes, à l'image du certificat d'aptitude professionnelle (CAP).

Au terme de ce travail, des acquis et des perspectives peuvent être évoqués. L'étude de la construction d'un système régional de formation en relation étroite avec les évolutions politiques et économiques que connaît le Nord permet sans doute de mieux comprendre les difficultés rencontrées au moment du défi de la reconversion dans la seconde moitié du $\mathrm{XX}^{\mathrm{e}}$ siècle - point sur lequel des études précises restent à mener. Les difficultés de la construction de ce territoire de la formation au travail montrent que la priorité accordée aux activités économiques locales n'a pas facilité la reconversion lorsque ces activités (textile et mines notamment) sont entrées en crise. Cela ne suffit pas à rendre compte des douleurs de la reconversion des années 1960, 1970 et 1980, mais entre en ligne de compte. De même, le constat répété de la domination des formations professionnalisantes courtes dans le paysage de la formation nordiste actuel trouve à l'évidence des origines historiques. Cette domination s'inscrit dans un rapport historiquement prudent des populations ouvrières et des catégories intermédiaires vis-à-vis des enseignements techniques - et l'observation mériterait d'être croisée avec l'étude du rapport à l'enseignement universitaire sur la longue durée.

Quant à la chronologie de l'institutionnalisation de la formation au travail, le cas de la région du Nord permet de confirmer une périodisation, qui va des conséquences spectaculaires de la première 
révolution industrielle jusqu'aux craquements d'un système progressivement édifié grâce en particulier aux piliers de la prospérité que furent le charbon et le textile. L'ensemble fonctionne sur le mode de la construction continuée, les remaniements de ce qui fonctionne ou de ce qui ne donne pas toute satisfaction étant fréquents, comme les tentations d'imiter ce qui semble fonctionner ailleurs. L'enchevêtrement des chronologies propres aux projets politiques nationaux et locaux intervient également pour offrir une institutionnalisation qui n'a rien de linéaire.

Trois temps de la formation se distinguent donc nettement: aux dispositifs anciens encore faiblement renouvelés, en raison de l'indifférence ou du rejet majoritaires (sans être unanimes) des formations, succède le passage à la formation comme enjeu de société de mieux en mieux identifié à partir des années 1880 . Si la chronique législative ne permet pas de rendre véritablement compte de l'action dans le domaine de l'enseignement technique, il existe bien une multiplication des initiatives et des réalisations, faiblement coordonnées. Il s'agit alors de former l'espace d'une véritable politique de l'enseignement technique - espace d'autant plus difficile à trouver que les différents partisans de la formation, bien qu'ils recourent tous à l'argument du besoin, ont des positions variées. La « crise de l'apprentissage » apparaît dès lors au moins autant comme une réelle période de doute que comme une opportunité pour ces partisans d'insister sur l'enjeu de la formation et de trouver des relais législatifs et économiques. La guerre marque une rupture. En obligeant à la coordination et à l'organisation des efforts, elle rend plus indispensable une politique de formation au travail, politique permise par les cadres réglementaire et financier nouveaux de la loi Astier (1919) et de la taxe d'apprentissage (1925). Dans les années 1920 et 1930, la territorialisation de la formation s'affirme, en lien avec la régionalisation économique même si elle ne s'y réduit pas.

Si le rapport entre les activités économiques et les dispositifs de formation est en définitive étroit, il ne se réduit pas à un rapport de cause à conséquence. Entre les activités économiques et les dispositifs de formation, cette recherche a l'ambition de rentrer dans la boîte noire des «besoins » de formation. Elle entend y repérer les intentions et les intérêts des responsables économiques, éducatifs et politiques, pour relever le défi de la complexité des logiques d'institutionnalisation de la formation au travail. Si celle-ci dans le Nord est le reflet des activités économiques régionales, encore faut-il nécessairement préciser, si l'on tente de poursuivre la métaphore, que le miroir est déformant.

Il est finalement tout sauf aisé d'étudier les rapports entre la formation professionnelle et les activités économiques. Ces rapports sont compliqués par les divergences d'intérêts, par l'expression difficile de ceux-ci, par le poids enfin des représentations économiques et sociales qui interviennent dans les débats et les décisions. On le voit, l'hypothèse néo-classique de la rentabilité de l'investissement dans la formation est plus récente et moins «naturelle» qu'on ne le suppose. En outre, les critiques à la recherche d'une faiblesse structurelle du système de formation et d'apprentissage français vont bien vite en besogne, aussi bien lorsqu'ils dénoncent le désintérêt ou la méconnaissance supposés des responsables éducatifs que lorsqu'ils manient l'école et l'économie comme deux entités étrangères l'une à l'autre ou homogènes l'une et l'autre.

Il me faut, pour terminer cet exposé, adresser de vifs remerciements, et d'abord à Jean-François Chanet, qui m'a laissé une grande liberté intellectuelle tout en se rendant toujours disponible pour faire progresser et aboutir cette recherche, et à Philippe Marchand, dont les travaux et l'intérêt pour cette thèse ont été précieux. J'adresse aussi des remerciements collectifs aux membres du jury, pour avoir bien voulu mettre cette thèse au nombre de leurs lectures estivales. 